\title{
The Perspectives of the General Population and Relatives of Cancer Patients with Respect to the Do-Not-Resuscitate Order
}

\author{
Ahmed H. Kaneetah ${ }^{1}$, Feras O. Baowaidan ${ }^{2}$, Bahaa A. Abulaban ${ }^{3}$, Mahmoud F. Sabban ${ }^{2}$, Ahmad S.
} Alshehri ${ }^{4}$

1. Internal Medicine, King Abdullah International Medical Research Center - King Saud Bin Abdulaziz University for Health Sciences, Jedah, SAU 2. Internal Medicine, King Abdullah International Medical Research Center - King Saud Bin Abdulaziz University for Health Sciences, Jeddah, SAU 3. Internal Medcine, King Abdullah International Medical Research Center - King Saud Bin Abdulaziz University for Health Sciences, Jeddah, SAU 4. Oncology, King Abdullah International Medical Research Center - King Saud Bin Abdulaziz University for Health Sciences, Jeddah, SAU

Corresponding author: Ahmed H. Kaneetah, kaneetahahmed@gmail.com

\section{Abstract \\ Background}

A do-not-resuscitate (DNR) order is a medical decision that instructs healthcare providers to withhold cardiopulmonary resuscitations (CPR) to patients in case of cardiopulmonary arrest in respect to their wishes. In Saudi Arabia, the decision is usually made by physicians based on the Fatwa number 12086 regardless of the patients' or their families' desires. Assessing the knowledge, perception, and attitude of Saudi family members towards this medical decision may help guide medical practitioners to make decisions that are legally and ethically acceptable for the patients and their family. Therefore, this study aimed to assess their knowledge, perception, and attitude about DNR decisions and to determine demographic variables that affect their attitude towards DNR decisions.

\section{Method}

This cross-sectional study was conducted from December 2017 to January 2018 utilizing survey distribution through emails and different social media outlets including Facebook, Twitter, Pinterest, and LinkedIn. A self-administered questionnaire was employed to elicit responses regarding knowledge, perception, and attitude towards DNR decisions. Statistical Package for the Social Sciences Windows version 17 (SPSS v.17) (IBM Corporation, USA) was used for data management and analysis.

\section{Result}

Of the 1882 participants who filled the questionnaire, only 1693 participants were eligible as the study sample population and were included in the final data analysis. Most of the participants were from the Makkah region (72.2\%) and were mainly from the general population (61.66\%). Participants were mainly females (66\%) and within the median age of 30 years (IQR: 21). As expected, participants who had heard about the DNR practice were from the medical field (58.6\%) and they were mainly distant relatives. Almost $76 \%$ of the participants had two to four incorrect answers about the DNR practice, and it indicated that participants have inadequate knowledge about a DNR order. Most of the participants (43.2\%) refused to be on a DNR order if they were diagnosed with a terminal illness. However, most participants (69.9\%) wanted to have an involvement in the decision-making of being on a DNR order. In terms of willingness to understand and learn about the DNR decision, 1475 (87.1\%) of the participants wanted to learn more about the DNR practice. Being a relative of a terminally ill cancer patient did not have a significant effect on the knowledge and perceptions of participants about being on a DNR order. However, having a background in medicine was found to be significantly associated with their acceptance to be on a DNR order. The knowledge score regarding DNR was found to be significantly associated with higher acceptance towards DNR orders.

\section{Conclusion}

The majority of participants had a lack of knowledge about the DNR practice. Their religion's concept of hope and virtue is considered as the major reason for their DNR order refusal. However, proper education about the DNR practice and involvement in the DNR order decision-making will increase the participants' knowledge and will improve their acceptance of the DNR practice.

Categories: Emergency Medicine, Family/General Practice, Oncology

Keywords: do not resuscitate order, dnr, saudi family, knowledge, acceptance

\section{Introduction}

A do-not-resuscitate (DNR) order is a medical decision that instructs healthcare providers to withhold 
cardiopulmonary resuscitations (CPR) to patients in case of cardiopulmonary arrest in respect to their wishes. It is usually suggested by physicians to terminally ill patients. However, in Saudi Arabia, the decision is usually made by physicians regardless of the patients' or their families' desires. This is according to the Fatwa number 12086 by the General Presidency of Scholarly Research and Ifta, which is an acceptable source of legislation. It also states that: "If three knowledgeable and trustworthy physicians agree that it is not appropriate to resuscitate a patient, when it is evident that they are suffering from an obstinate, incurable illness and that death is inevitable, there is no need to use resuscitators, life-supporting machines" [1].

Multiple studies in the literature have shown that demographic variables such as age, race, religion, marital status, and educational level are associated with different knowledge, perception, and attitudes towards DNR orders [2-6]. Thus, a local study is needed to assess the knowledge, perception, and attitudes towards DNR orders in Saudi Arabia.

DNR orders are often questioned on ethical and religious grounds because 1) it appears as if physicians are letting their patients die, and 2) even if they resuscitate the patient and let the patient survive, the resuscitation will not cure the disease; this also sometimes leads to more complications that prolong the patients' suffering. According to the American Heart Association (AHA) report in 2016, the success rate of CPR in the general population despite their medical illness is $24.8 \%$ inside hospitals and much less outside [7]. According to a systematic review, the chance of survival to discharge after CPR for advanced cancer patients is $10.1 \%$ if the resuscitation occurs in the wards, and $2.2 \%$ if it occurs in intensive care units (ICUs) [8]. Moreover, the return of spontaneous circulation (ROSC) is lower in patients with cancer [9] and most of those with advanced cancer in whom CPR was successful die within days to weeks in the ICU [10-13].

Previous studies in Saudi Arabia have evaluated knowledge and attitude of outpatients, interns, and residents towards DNR orders [14-16]. However, there is no study that assessed the knowledge, perception, and attitudes towards DNR orders among the general population as well as cancer patients' relatives in Saudi Arabia. In addition, there are no studies in Saudi Arabia that evaluated how demographic variations can affect the knowledge, perception, and attitudes towards DNR orders.

Relatives of cancer patients are the ones who usually witness and experience the DNR practices in hospitals. Assessing their knowledge, perception, and attitude towards this medical decision may help guide medical practitioners to make decisions that are legally and ethically acceptable for the patients and their families. Therefore, this study aimed to assess their knowledge, perception, and attitudes about DNR decisions and to determine demographic variables that affect attitudes towards DNR decisions.

\section{Materials And Methods}

This cross-sectional study was conducted from December 2017 to January 2018. It aimed to determine the perspectives of the general population and relatives of cancer patients with respect to the DNR order. Respondents were recruited nationally and internationally through invitations in emails and different social media outlets including Facebook, Twitter, Pinterest, and LinkedIn. Eligible Saudi respondents were those aged 18 years old and above, as well as who had experienced or witnessed a family relative diagnosed with terminal cancer. Non-Saudi and those have been diagnosed with cancer were excluded.

A self-administered questionnaire developed by the authors was employed. The face and construct validity of the questionnaire was addressed through consultation with three oncology and two intensive care consultants, as well as with several family members and relatives of the terminally 111 cancer patients in Princess Noorah Oncology Center, King Abdulaziz Medical City, Jeddah, Saudi Arabia. Individual items were evaluated based on their relevance to DNR content domain. Items were also evaluated based on their affective and cognitive representativeness to the target population of this study. Thus, the final questionnaire developed consisted of three parts. The first part dealt with the demographical data of the respondents, which included questions regarding their age, gender, marital status, region, income, educational level, employment, and medical background (i.e. student or work related to medicine). The respondents were also asked if they had family members who had ever been diagnosed with cancer and whether they had heard about the DNR practice before. The second part consisted of seven multiple-choice questions that assessed the respondents' knowledge regarding the concept of DNR. The third part consisted of five questions that assessed the attitude of respondents towards DNR decisions. It included questions that asked the respondents about their willingness to be on a DNR order if they were diagnosed with a terminal illness and if they wished to be involved in the DNR decision-making. It also included questions to assess the respondents' willingness to discuss DNR decisions and learn more about it.

All data collected were grouped according to the degree of family relationship with regard to the terminally ill cancer patient. First-degree relatives included parents, siblings, children, and spouses. Second-degree relatives included grandparents, grandchildren, uncles, aunts, first cousins, nephews, and nieces. Distant relatives included relatives who are not first- or second-degree relatives. General populations were those who did not have any relation to the cancer patient. 


\section{Cureus}

data management and analysis. Descriptive analysis, standard deviations, and percentages were performed for normally distributed variables, while mean and interquartile range (IQ) were used for skewed distributed variables. To determine significant associations between categorical variables, the chi square test was used. The Kruskal-Wallis test was used to test for differences in the knowledge among the different subgroups regarding the DNR practice. Cross tabulation was done to compare attitudes towards the DNR decisions among the different subgroups. Lastly, Pearson's regression analysis was employed to determine confounding factors. Statistical significance was set at $\mathrm{p}<0.05$.

\section{Results}

\section{Demographic data}

Of the total 2830 Saudi survey participants invited, 1882 (i.e. $66.50 \%$ response rate) of them filled the questionnaire. However, those who did not meet the eligibility criteria and those who did not completely fill the questionnaire were excluded. Thus, only a total of 1693 respondents were included in the final analysis.

Analysis showed that most of the respondents were females (66\%) and mainly within the median age of 30 years (IQR: 21). Respondents were from the 13 administrative regions of Saudi Arabia and most of them were from the Makkah region (72.2\%). Respondents mostly had a bachelor's degree $(51.1 \%)$ and were in nonmedical fields (67.6\%).

The study sample population comprised of 281 (16.5\%) first-degree relatives of the cancer patient, 327 (19.3\%) were second-degree relatives, 33 (1.9\%) were distant relatives, and 1052 (61.66\%) were from the general population. The complete details of the respondents' demographics for each group are presented in Table 1. 


\begin{tabular}{|c|c|c|c|c|c|c|}
\hline & & \multirow{2}{*}{ All } & \multicolumn{4}{|c|}{ Degree of Family Relationship } \\
\hline \multicolumn{2}{|c|}{ Demographic Characteristics } & & $1^{\text {st }}$ Degree & $2^{\text {nd }}$ Degree & Distant Relative & General Population \\
\hline & & Median (IQR) & Median (IQR) & Median (IQR) & Median (IQR) & Median (IQR) \\
\hline Age & & $30(21)$ & $42(20)$ & $25(17)$ & $23(13)$ & $29(18)$ \\
\hline Gender & Female & $1129(66.7)$ & $191(68)$ & $250(76.5)$ & $23(69.7)$ & 656 (63.2) \\
\hline \multirow{2}{*}{ Marital status } & Married & $871(51.5)$ & $188(66.9)$ & $141(43.1)$ & $11(33.3)$ & $531(50.5)$ \\
\hline & Not married & $821(48.5)$ & $93(33.1)$ & $186(56.9)$ & $22(66.7)$ & $520(49.5)$ \\
\hline \multirow{7}{*}{ Region } & Makkah & 1218 (72.2) & 209 (74.4) & $234(72.2)$ & $27(81.8)$ & $748(71.3)$ \\
\hline & Riyadh & $161(9.5)$ & $21(7.5)$ & $30(9.3)$ & $1(3)$ & 109 (10.4) \\
\hline & Eastern province & $129(7.6)$ & $26(9.3)$ & $36(11.1)$ & $4(12.1)$ & $63(6)$ \\
\hline & Madinah & $52(3.1)$ & $9(3.2)$ & $8(2.5)$ & 00 & $35(3.3)$ \\
\hline & Asir & $43(2.5)$ & $5(1.8)$ & $5(1.5)$ & 00 & $33(3.1)$ \\
\hline & Other regions & $72(4.3)$ & $9(3.2)$ & $10(3.1)$ & $1(3)$ & $52(5)$ \\
\hline & Outside & $12(.7)$ & $2(.7)$ & $1(.3)$ & 00 & $9(.9)$ \\
\hline \multirow{2}{*}{ Income } & $\leq 10000$ & $714(42.2)$ & $111(39.5)$ & $135(41.3)$ & $12(36.4)$ & 456 (43.3) \\
\hline & $>10000$ & $979(57.8)$ & $170(60.5)$ & $192(58.7)$ & $21(63.6)$ & $596(56.7)$ \\
\hline \multirow{5}{*}{ Educational level } & & $28(1.7)$ & $8(2.9)$ & $3(.9)$ & 00 & $17(1.6)$ \\
\hline & High school & $486(28.7)$ & $47(16.8)$ & $119(36.4)$ & $17(51.5)$ & $303(28.8)$ \\
\hline & Diploma & $156(9.2)$ & $27(9.6)$ & $24(7.3)$ & $1(3)$ & $104(9.9)$ \\
\hline & Bachelor & $864(51.1)$ & $155(55.4)$ & $153(46.8)$ & $12(36.4)$ & $544(51.7)$ \\
\hline & Higher education & $158(9.3)$ & $43(15.4)$ & $28(8.6)$ & $3(9.1)$ & $84(8)$ \\
\hline \multirow{4}{*}{ Employment } & Employed & $733(43.4)$ & $141(50.5)$ & $119(36.4)$ & $10(30.3)$ & $463(44.1)$ \\
\hline & Not empioyed & $237(14)$ & $38(13.6)$ & $51(15.6)$ & $3(9.1)$ & $145(13.8)$ \\
\hline & Student/trainee & $569(33.7)$ & $42(15.1)$ & $136(41.6)$ & $18(54.5)$ & $373(35.5)$ \\
\hline & Retire & $151(8.9)$ & $58(20.8)$ & $21(6.4)$ & $2(6.1)$ & $70(6.7)$ \\
\hline \multirow{3}{*}{ Specialty } & Medical & $548(32.4)$ & $64(22.8)$ & $133(40.7)$ & $10(30.3)$ & 341 (32.4) \\
\hline & Non-medical & $1145(67.6)$ & $217(77.2)$ & $194(59.3)$ & $23(69.7)$ & 711 (67.6) \\
\hline & Total & 1693 & 281 & 327 & 33 & 1052 \\
\hline
\end{tabular}

TABLE 1: Participants' demographic characteristics.

\section{Knowledge about DNR}

We found that 541 (32\%) respondents had heard about the DNR order before. As expected, most of them were from the medical field (58.6\%) as compared to a non-medical field (19.2\%). The majority were distant relatives (45.5\%) followed by second-degree relatives (38.8\%), general population (30.5\%), and first-degree relatives (27.8\%). The items that assessed DNR knowledge found that majority (54\%) of the respondents answered four or five questions correctly, 25.4\% answered six or seven questions correctly, and $20.6 \%$ answered three questions or fewer correctly. Almost $76 \%$ of the respondents had two to four incorrect answers about the DNR practice. The median knowledge score was five out of seven. However, Table 2 shows that having a relative with cancer doesn't have an influence on the DNR knowledge score $(\mathrm{p}=0.649)$. The only 


\section{Cureus}

demographic variable that was associated with better knowledge score is being in a medical field ( $\mathrm{p}=0.003)$ (Table 3).

\begin{tabular}{|c|c|c|c|}
\hline \multicolumn{2}{|c|}{ Degree of Family Relationships } & $n(\%)$ & Kruskal-Wallis $P$ value \\
\hline General & & $1052(61.66)$ & \multirow{4}{*}{0.649} \\
\hline \multirow{3}{*}{ Relatives } & $1^{\text {st }}$ & $281(16.5)$ & \\
\hline & $2^{\text {nd }}$ & $327(19.3)$ & \\
\hline & $3^{\text {rd }}$ & $33(1.9)$ & \\
\hline
\end{tabular}

TABLE 2: Knowledge score when grouped according to degree of family relationships.

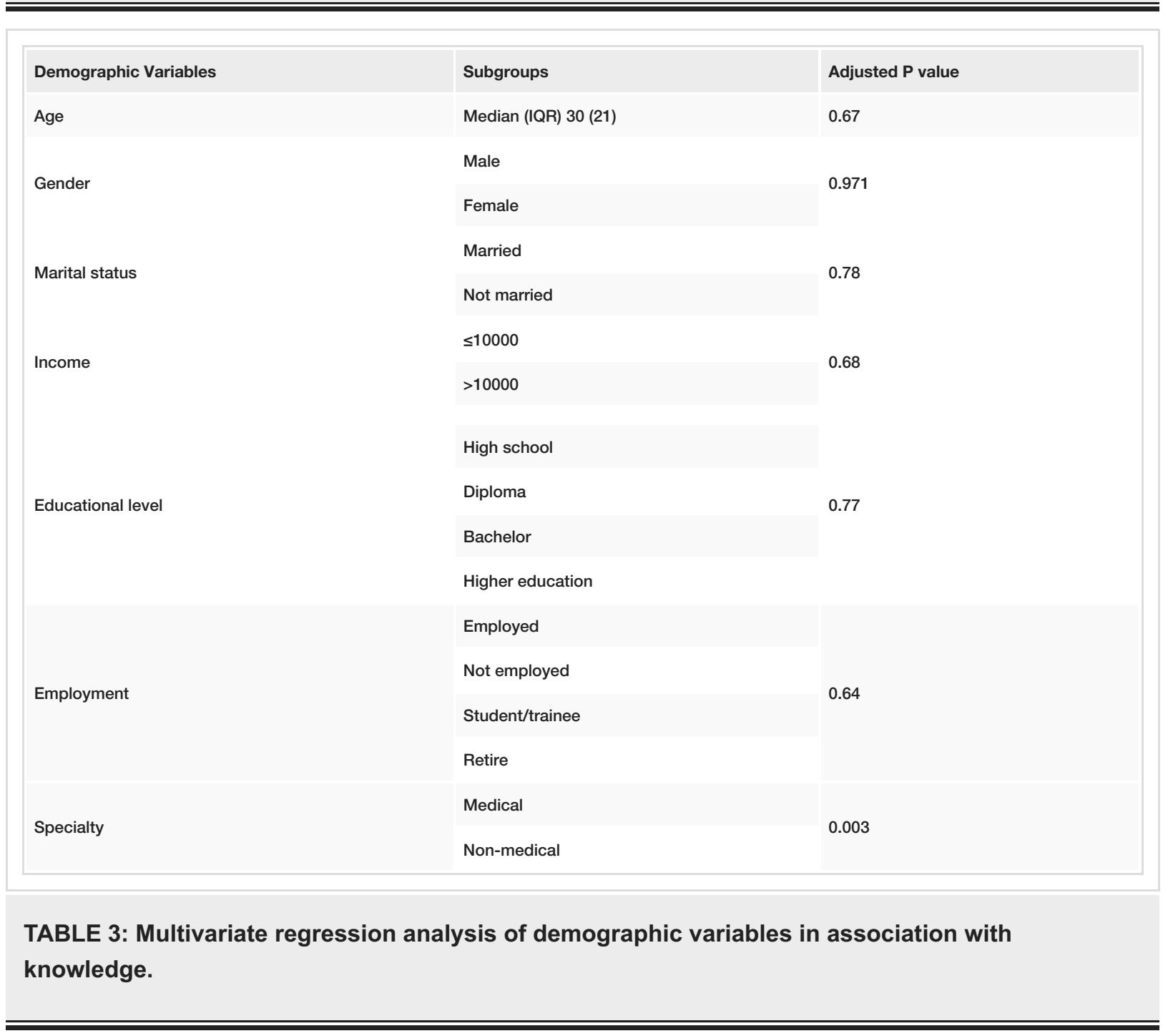

\section{Attitude towards DNR}

Most of the respondents (43.2\%) refused to be on a DNR order if they were diagnosed with a terminal illness. The most common reasons of refusal were of hope and religious concern. Other reasons were related to their responsibilities and family-related concerns.

Most respondents (69.9\%) wanted to be involved in the decision-making of being on a DNR order. Considering life situation or circumstances, there were 1229 (72.6\%) respondents who wanted to discuss DNR orders while they were in good health, 269 (15.9\%) wanted to discuss it only if they were diagnosed with a terminal illness, and 195 (11.5 \%) did not want to discuss it at all. In terms of willingness to understand and learn about DNR decisions, there were 1475 (87.1\%) respondents who wanted to learn more 


\section{Cureus}

about the DNR practice. Being a cancer patient's relative did not affect their preference to be on a DNR order (Table 4). Furthermore, demographic variations were found not associated with any change in acceptance of being on a DNR order except for being in a medical field. Being in a medical field is associated with higher acceptance of being on a DNR order $(\mathrm{p}=0.002)$ (Table 5).

\begin{tabular}{|c|c|c|c|c|c|}
\hline & & \multicolumn{3}{|c|}{ If I were diagnosed with a terminal illness, I prefer to be on DNR. } & \multirow{2}{*}{ P Value } \\
\hline & & Agree (\%) & Neutral (\%) & Disagree (\%) & \\
\hline \multicolumn{2}{|l|}{ General } & $294(27.9)$ & $298(28.3)$ & $460(43.7)$ & \multirow{5}{*}{0.27} \\
\hline \multirow{3}{*}{ Relatives } & $1^{\text {st }}$ degree & $93(33.1)$ & $71(25.3)$ & $117(41.6)$ & \\
\hline & $2^{\text {nd }}$ degree & $108(33)$ & $82(25.1)$ & $137(41.9)$ & \\
\hline & Distant & $6(18.2)$ & $9(27.3)$ & $18(54.5)$ & \\
\hline All & & $501(29.6)$ & $460(27.2)$ & $732(43.2)$ & \\
\hline
\end{tabular}

TABLE 4: Acceptance towards DNR when grouped according to degree of relatives.

\begin{tabular}{|c|c|c|c|c|c|}
\hline & & \multicolumn{4}{|c|}{ If I were diagnosed with a terminal illness, I prefer to be on DNR } \\
\hline & & Agree (\%) & Neutral (\%) & Disagree (\%) & sig \\
\hline \multirow{2}{*}{ Specialty } & Medical & $186(33.9)$ & $158(28.8)$ & 204 (37.2) & \multirow{2}{*}{0.002} \\
\hline & Non- medical & $315(27.5)$ & $302(26.4)$ & $528(46.1)$ & \\
\hline
\end{tabular}

TABLE 5: Acceptance towards DNR between medical and non-medical respondents.

\section{Association between knowledge and attitude towards DNR}

Lastly, this study found that knowledge score is significantly associated with high acceptance towards the DNR order ( $\mathrm{p}=0.007)$ (Table $)$.

\begin{tabular}{|c|c|c|c|}
\hline & n (\%) & Mean Rank & Kruskal-Wallis $\mathbf{P}$ value \\
\hline Agree & $501(29.6)$ & 888.69 & \\
\hline Neutral & $460(27.2)$ & 865.95 & 0.007 \\
\hline Disagree & 732 (43.2) & 806.56 & \\
\hline
\end{tabular}

TABLE 6: Association between knowledge and acceptance towards DNR.

\section{Discussion}

The DNR decision of patients is legal in some countries and prohibited or restricted in some other countries. In addition, implementation of DNR orders is a controversial issue. Do we harm patients with DNR? Some studies have presented the effects of DNR on the ROSC and results of CPR among patients with malignancy and end-stage disease $[9,17]$ and therefore consider the DNR order as an acceptable option for patients with terminal cancer. On the other hand, DNR orders are usually questioned because they can be perceived as antireligious or ethically unacceptable. Saudi Arabia is one of the most religious countries in the world and the distinct Saudi culture also has different ethical and moral standards compared to those of other parts of the world. These differences have a great impact on the knowledge, perception, and attitude towards very sensitive topics such as DNR. In our study $32 \%$ of all respondents have heard about the DNR practice before. This is much less than another study done in outpatient clinics in Riyadh, Saudi Arabia in 2017 and a Canadian study done in primary care centers in North Vancouver in 2009. The percentage of those who have heard about the term DNR before were $75 \%$ and $84 \%$, respectively $[4,16]$. Contrary to what we hypothesized, 
there is no association between the relationship with the patient and having heard about DNR before. There is no study in the related literature that investigates this association. As expected, those who study or work in the medical field have heard more about DNR orders (58.6\%) in comparison to those who did not study or work in the medical field (19.2\%). One study done among interns and residents in Jeddah, Saudi Arabia in 2016 showed that $92 \%$ of residents and $70 \%$ of interns were familiar with the term DNR [14]. In another study in Hong Kong in 2007, 66.4\% of non-medical students and $18.7 \%$ of medical students had never heard of DNR [18]. What could explain the lower percentages in our study is that we included all those who study or work in any medical field that might not be exposed to DNR orders.

Having a relative with cancer was not associated with better knowledge about DNR. This can be explained by the fact that DNR is a unilateral decision in Saudi Arabia. Therefore, physicians do not explain the DNR practice properly to their patients and the relatives, which might lead to insufficient hospital education. Only a single study in Canada with nine respondents in a care givers' group has assessed the understanding regarding the term DNR, and six out of the nine respondents showed good knowledge [19].

In a study in the United States among inpatients admitted for general medical services, $16 \%$ refused to be on DNR if they were diagnosed with a terminal illness in comparison to $43.2 \%$ of the respondents [5]. The most common reasons for refusal in the said study were hope, religious reasons, or both. It is also worth noting that although the decision in Saudi Arabia is solely made by physicians, most of the respondents wanted to be involved in the decision-making.

According to a study done in Saudi Arabia among outpatients, 90\% of the respondents wanted to discuss DNR while they were ill and $10 \%$ while they were healthy [16]. In another study in Canada, $56 \%$ of the respondents preferred to discuss the matter when they were still healthy [4]. Interestingly, $72.6 \%$ of the respondents wanted to discuss DNR orders while they are in good health, $15.9 \%$ wanted to discuss it only if they are diagnosed with a terminal illness, and $11.5 \%$ did not want to discuss it at all. Having a relative with cancer does not play a significant role in the preference of being on a DNR order. As expected, having more knowledge is associated significantly with higher acceptance. In addition, most respondents wanted to learn more about DNR orders. Consequently, more education is needed in the hospital setting regarding this matter as most are keen to learn more about this topic, which can improve the acceptance rate. In addition, physicians might consider discussing these topics with their patients when they are still healthy.

\section{Conclusions}

The majority of participants had a lack of knowledge about the DNR practice. The concept of hope and virtue of their religion were considered as the major reasons for DNR refusal. However, being in a medical field is a significant factor in understanding the concept of DNR acceptance. The study only goes to show that educating family members and relatives about DNR and involving them in the DNR decision-making is essential to improve their knowledge and acceptance of DNR orders.

\section{Additional Information \\ Disclosures}

Human subjects: Consent was obtained by all participants in this study. Animal subjects: All authors have confirmed that this study did not involve animal subjects or tissue. Conflicts of interest: In compliance with the ICMJE uniform disclosure form, all authors declare the following: Payment/services info: All authors have declared that no financial support was received from any organization for the submitted work. Financial relationships: All authors have declared that they have no financial relationships at present or within the previous three years with any organizations that might have an interest in the submitted work. Other relationships: All authors have declared that there are no other relationships or activities that could appear to have influenced the submitted work.

\section{Acknowledgements}

This research was supported by the Research Unit of the College of Medicine, King Saud Bin Abdulaziz University for Health Sciences, Jeddah. We thank Dr. Basim Alsaywid who provided his insights and expertise that greatly made this research possible. We would also like to thank Dr. Mohammad Khan and Juan S. Manlangit Jr. for helping us with our research methodology and statistical treatment. Lastly, we would like to show our gratitude to Dr. Faisal Boker for his clever ideas and continuous support during the course of this research.

\section{References}

1. Fatwas on medical Issues and the sick: resuscitating a dead or incurable patient who is not fit for resuscitation under Fatwa no. 12086 . (1989). Accessed: July 14, 2018: http://www.alifta.net/Fatawa/FatawaChapters.aspx? languagename $=$ en $\&$ View=Page $\&$ PageID $=299 \&$ PageNo=1\&BookID $=17$.

2. Garrido MM, Harrington ST, Prigerson HG: End-of-life treatment preferences: a key to reducing ethnic/racial disparities in advance care planning?. Cancer. 2014, 120:3981-3986. 10.1002/cncr.28970 
3. Hileli I, Weyl Ben Arush M, Hakim F, Postovsky S: Association between religious and socio-economic background of parents of children with solid tumors and DNR orders. Pediatr Blood Cancer. 2014, 61:265268. 10.1002/pbc. 24712

4. Robinson C, Kolesar S, Boyko M, Berkowitz J, Calam B, Collins M: Awareness of do-not-resuscitate orders: what do patients know and want?. Can Fam Physician. 2012, 58:229-233.

5. Frankl D, Oye RK, Bellamy PE: Attitudes of hospitalized patients toward life support: a survey of 200 medical inpatients. Am J Med. 1989, 86:645-648. 10.1016/0002-9343(89)90436-1

6. Zaide GB, Pekmezaris R, Nouryan CN, et al.: Ethnicity, race, and advance directives in an inpatient palliative care consultation service. Palliat Support Care. 2013, 11:5-11. 10.1017/S1478951512000417

7. CPR Facts and Stats: How CPR is changing (and saving) lives . (2017). Accessed: December 21, 2018: http://cpr.heart.org/AHAECC/CPRAndECC/General/UCM_477263_Cardiac-Arrest-Statistics.jsp.

8. Reisfield GM, Wallace SK, Munsell MF, Webb FJ, Alvarez ER, Wilson GR: Survival in cancer patients undergoing in-hospital cardiopulmonary resuscitation: a meta-analysis. Resuscitation. 2006, 71:152-160. 10.1016/j.resuscitation.2006.02.022

9. Gulacti U, Lok U, Aydin I, et al.: Outcomes of in-hospital cardiopulmonary resuscitation after introduction of medical emergency team. Kuwait Med J. 2016, 48:127-131.

10. Ahmad AS, Mudasser S, Khan MN, Abdoun HN: Outcomes of cardiopulmonary resuscitation and estimation of healthcare costs in potential 'do not resuscitate' cases. Sultan Qaboos Univ Med J. 2016, 16:27-34. 10.18295/squmj.2016.16.01.006

11. Kjørstad OJ, Haugen DF: Cardiopulmonary resuscitation in palliative care cancer patients. [Article in English, Norwegian]. Tidsskr Nor Laegeforen. 2013, 133:417-21. 10.4045/tidsskr.12.0378

12. Fu S, Hong DS, Naing A, et al.: Outcome analyses after the first admission to an intensive care unit in patients with advanced cancer referred to a phase I clinical trials program. J Clin Oncol. 2011, 29:3547-3552. 10.1200/jco.2011.29.15_suppl.e13020

13. Loertscher L, Reed DA, Bannon MP, Mueller PS: Cardiopulmonary resuscitation and do-not-resuscitate orders: a guide for clinicians. Am J Med. 2010, 123:4-9.

14. Amoudi AS, Albar MH, Bokhari AM, Yahya SH, Merdad AA: Perspectives of interns and residents toward donot-resuscitate policies in Saudi Arabia. Adv Med Educ Pract. 2016, 7:165-170. 10.2147/AMEP.S99441

15. Aljohaney A, Bawazir Y: Internal medicine residents perspectives and practice about do not resuscitate orders: survey analysis in the western region of Saudi Arabia. Adv Med Educ Pract. 2015, 6:393-398. 10.2147/AMEP.S82948

16. Al Sheef MA, Al Sharqi MS, Al Sharief LH, Takrouni TY, Mian AM: Awareness of do-not-resuscitate orders in the outpatient setting in Saudi Arabia. Perception and implications. Saudi Med J. 2017, 38:297-301. 10.15537/smj.2017.3.18063

17. Gulacti U, Lok U: Influences of "do-not-resuscitate order" prohibition on CPR outcomes . Turk J Emerg Med. 2016, 16:47-52. 10.1016/j.tjem.2016.03.003

18. Sham CO, Cheng YW, Ho KW, et al.: Do-not-resuscitate decision: the attitudes of medical and non-medical students. J Med Ethics. 2007, 33:261-5. 10.1136/jme.2005.014423

19. Ahmed N, Lobchuk M, Hunter WM, et al.: How, when and where to discuss do not resuscitate: a prospective study to compare the perceptions and preferences of patients, caregivers, and health care providers in a multidisciplinary lung cancer clinic. Cureus. 2015, 7:257. 10.7759/cureus.257 